\title{
MICROBIAL BIOBURDEN OF ORTHOKERATOLOGY AND HYDROGEL CONTACT LENSES AND STORAGE CASES USING A HYDROGEN-PEROXIDE DISINFECTING SYSTEM: A PILOT STUDY
}

Ananya Datta BOPTOM MPhil PhD FAAO, Erin S. Tomiyama OD MS FAAO,

Kathryn Richdale OD PhD FAAO

University of Houston College of Optometry, Texas, USA

Corresponding Author: Kathryn Richdale email: richdale@central.uh.edu

Submitted: 8 May 2021. Accepted: 9 September 2021. Published: 26 October, 2021.

\begin{abstract}
Background and objective: The fitting of orthokeratology (ortho-k) and multifocal soft contact lenses (SCL) is becoming increasingly popular worldwide for reducing the rate of myopia progression. However, overnight wear use of lenses and microbial contamination of contact lenses and storage vials has been shown to increase the risk of corneal inflammatory and infiltrative events (CIEs). Therefore, we conducted a pilot study to compare the rate, level, and type of microbial contamination of contact lenses and storage vials when participants wear ortho-k and SCL in combination with a hydrogen-peroxide disinfecting system.

Material and Methods: A prospective, single-centre, randomized, crossover study was conducted to evaluate the rate and level of contact lens and storage vials contamination during the use of ortho-k and SCL along with a hydrogen-peroxide disinfecting solution over two 10 day wearing periods. Ocular signs and symptoms were assessed during lens wear at baseline and after each wearing period. In addition, contact lens and storage vials were collected, and the type and amount of microbial contamination were evaluated using viable culture and standard identification methods.

Results: Twenty-five adults were enrolled, and 21 completed the study. One (5\%) ortho-k lens, five (24\%) ortho-k storage vials, one (5\%) SCL and one (5\%) SCL storage vial were contaminated (P > 0.05), predominantly with Gram-positive bacteria. None of the participants had contamination with both ortho-k and SCL. No significant differences were found between the ocular signs, including conjunctival redness and roughness, or conjunctival or corneal staining (all $\mathrm{P}>0.05$ ), irrespective of using ortho-k or SCL.

Conclusion: This study provides the first data that directly compares microbial contamination of ortho$\mathrm{K}$ versus SCL in patients using a hydrogen-peroxide disinfection system. However, the overall microbial contamination rate of contact lens storage vials was much lower (30\%) than the previously reported study
\end{abstract}

J Cont Lens Res Sci Vol 5(1):e19-e31; October 26, 2021

This article is distributed under the terms of the Creative Commons Attribution-

Non Commercial 4.0 International License. @Datta et al. 
with ortho-k lens wear. The results support conducting a larger clinical trial designed to understand differences in microbial contamination with different lens materials and modalities.

Keywords: orthokeratology, soft contact lens, lens storage case, microbial contamination, overnight lens wear.

\section{INTRODUCTION}

Since the early $19^{\text {th }}$ century, contact lenses have been used as an alternative to spectacles to correct refractive error, therapeutic treatment, or cosmesis. Globally, there are 140 million people who wear contact lenses. ${ }^{1}$ Of the $45+$ million contact lens wearers in the United States, ${ }^{1} 88 \%$ wear soft contact lenses (SCL) and approximately $10-12 \%$ rigid lenses, including orthokeratology (ortho-k). ${ }^{1,2}$ Ortho-k lenses are reverse geometry corneal reshaping rigid contact lenses. They are becoming an increasingly popular, well-accepted myopia management option to reduce the rate of myopia progression. ${ }^{3-6}$ However, the use of lenses overnight causes an increased concern for contact lens induced corneal infection and microbial keratitis (MK), compared to daily lens wear. ${ }^{7-12}$

Complications associated with SCL and ortho$\mathrm{k}$ lenses include corneal epithelial defects, corneal edema, and corneal infiltrative and infectious events, including MK. ${ }^{13,14}$ Whilst rare, ${ }^{15} \mathrm{MK}$ is serious, potentially blinding corneal infection ${ }^{16,17}$ and overnight contact lens wear is a significant risk factor. ${ }^{8,9,11,18}$ Contact lens acute red eye (CLARE) is, by definition, associated with closed eye lens wear (e.g., napping or sleeping in lenses). ${ }^{19,20}$ CLARE is most commonly observed during the first three months of extended wear, although it can occur at any time. ${ }^{21,22}$ Risk factors associated with CLARE include high water content soft lenses, a tight-fitting lens, and patients who suffer from respiratory tract infection. ${ }^{23}$ Contact lens-induced peripheral ulcer (CLPU) is another condition often associated with extended wear contact lens use, as Szczotka-Flynn et al (2013) reported. ${ }^{24}$ The incidence of CLPU was always higher with extended wear asymptomatic (extended wear vs. daily wear: up to $25 \%$ vs. up to $20 \%$ ) or symptomatic (extended wear vs. daily wear: up to $6 \%$ vs. up to $3 \%$ ) contact lens wear compared to daily wear, even with the use of silicone hydrogel contact lenses. ${ }^{24}$

Multiple genera and species are associated with contact lens induced MK, including Pseudomonas aeruginosa, ${ }^{25-28}$ Staphylococcus aureus, ${ }^{27}$ Acanthamoeba species, ${ }^{27-31}$ and fungi especially Fusarium spp. Aspergillus spp. and Candida spp. have also been isolated but at a much lower frequency than other bacteria. ${ }^{8,18,27,32}$ The causative microorganisms have been isolated from contact lenses and contact lens related accessories, particularly from lens storage vials and disinfecting solutions. ${ }^{11}$ Contact lens storage cases are the most commonly contaminated contact lens accessories ${ }^{33}$, and they are a risk factor for the development of contact lens induced MK. ${ }^{12}$

Microbial contamination of contact lenses and storage cases varies depending upon the types of lens materials, wearing modality, cleaning solution, and behavioural use factors. ${ }^{12,34-37}$ Hydrogen-peroxide solution has been shown to reduce the rates of MK and other corneal infiltrative events compared to multipurpose disinfecting solutions (MPDS) ${ }^{38}$ To date, no study has examined the type and amount of contamination in patients fitted with both ortho-k and SCLs. Therefore, this pilot study aimed to compare the level (total contamination) and rate (number of colony-forming units) of microbial contamination and the type of microorganisms recovered from contact lenses and lens storage cases for ortho-k and SCLs used with a hydrogen-peroxide disinfecting system as part of a larger crossover clinical trial.

\section{METHODS}

This study was a part of a more extensive prospective, randomized, crossover, single centre study conducted at the University of Houston College of Optometry. The preliminary study explored changes in higher order aberrations and peripheral refraction in myopic astigmats fitted with soft multifocal toric contact lenses 
and toric orthokeratology lenses. ${ }^{39}$ To date, one paper on the higher-order aberration outcomes has been published, and others are still in development. ${ }^{39}$ As a secondary outcome, microbial contamination was explored to determine the necessity of a larger trial. Twenty-five participants were enrolled, and microbial samples were available from 21 participants who completed all five study visits. A literature review was done for the microbial contamination of contact lens and lens storage cases/vials. Based on the previous literature, the range of soft lens contamination varied between $25 \%$ to $92 \%$, and for the ortho-k and rigid gas permeable lenses, contamination varied between $1 \%$ to $38 \%$. We have considered the minimum level of lens/ lens storage case contamination for the sample size calculation. $^{26,34,36-38,40-47}$ This sample was estimated at the $5 \%$ level of significance and with $80 \%$ power and assuming a $10 \%$ dropout rate to demonstrate the type and amount of contact lens and storage case contamination. Due to the crossover study design, each participant served as their own control, permitting paired statistical testing to evaluate differences.

\section{Recruitment and entry criteria}

The study protocol was submitted for ethics approval by the University of Houston (UH) Institutional Review Board (IRB) and was approved and registered on ClinicalTrials.gov before enrolment of any participants (NCT \#03728218). Potential participants were recruited from the patients, faculty, students, and staff of the University Eye Institute at the UH College of Optometry, as well as the surrounding community via verbal communication, print media (e.g., study flyers), telephone and electronic media (e.g., email, social media). Adult participants, 18 to 39 years of age, with normal ocular health and no contraindications to contact lens wear were recruited. Eligible participants had a vertexed corrected sphere power between plano to -5.00 diopters (D) and vertexed refractive cylinder power between -1.50 to $-3.50 \mathrm{D}$ in each eye. Participants had a best-corrected Snellen acuity of $6 / 7.5$ or better in each eye and had no history of ocular pathology or surgery, active ocular infection, or clinically significant ocular inflammation. Participants must not have had any significant binocular vision abnormalities, worn gas permeable contact lenses for at least one month, nor be pregnant or nursing (by self-report).

\section{Study devices}

The commercially available and United States Food and Drug Administration (FDA) approved soft multifocal toric contact lenses (Proclear Multifocal Toric; CooperVision ${ }^{\circledR}$, San Ramon, CA, USA) and toric periphery ortho-k (Paragon CRT ${ }^{\circledR}$ Dual Axis Contact Lenses; Paragon Vision Sciences, Gilbert, AZ, USA) contact lenses were used for this study. Lenses were ordered based on the manufacturer's fitting guides and CLEAR CARE® (Alcon, Fort Worth, TX, USA) 3\% hydrogen peroxide solution was provided for disinfecting and storage of both types of contact lenses. All participants were instructed to follow the manufacturer's provided contact lens cleaning regimens, including avoiding water exposure to the contact lens or lens storage vial and not use any other solutions or eye drops during the study. SCLs were worn daily, and ortho-k lenses were worn overnight in a randomized order, each for $10 \pm 2$ days/nights and at least seven hours per day/night.

\section{Study design}

Each participant was seen for a total of five visits over two months approximately. Visit 1 was a baseline evaluation, where both types of contact lens were ordered empirically. Visit 2 was a contact lens fitting of both lens types and randomization to the first lens. Visit 3 was an outcome visit for the first lens. Visit 4 occurred after a $14 \pm 2$-day washout period, where the participants were dispensed the second lens. Lastly, Visit 5 was an outcome visit for the second lens. All outcome visits were conducted between noon and $6 \mathrm{pm}$. Of importance to this arm of the study, an extra pair of contact lenses were made to dispense unworn lenses at Visit 4 since both lens types were applied for fit assessment at Visit 2.

Participants were responsible for wearing the assigned contact lenses for at least seven hours every day for SCL or every night ${ }^{48}$ for ortho-k lenses between study visits 2 - 3 and 4 - 5. In addition, participants were instructed to wear the SCL for at least two hours before coming to the outcome study visit. After $10 \pm 2$ days of each lens wear (Visits 3 and 5), study lenses and lens storage vials were collected and stored for microbial sample collection. Contact lenses were removed by the 
participant and placed in the lens vial either the morning before the visit (ortho-k) or at the visit (SCL). At the end of the $3^{\text {rd }}$ and $5^{\text {th }}$ visits, participants were also asked to complete a questionnaire regarding ocular comfort, subjective vision, and lens handling using the Ranked Symptoms Scale (RSS) survey. ${ }^{49}$ Slit lamp findings were assessed at every visit using the Cornea and Contact Lens Research Unit (CCLRU) grading scale to evaluate the type and extent of corneal staining.

\section{Microbial sample collection and analysis}

All procedures involving the microbial samples were conducted within the microbiology laboratory, which has safety procedures to minimize infectious and other potential risks to personnel. On the study visit, participants placed their lenses in their storage vials with the disinfecting solution, as they usually would. To assess microbial contamination lenses and storage vials were sent to the microbiology laboratory within one hour of collection. A log-sheet was maintained of the collection and process time to ensure that all lenses remained in the hydrogen-peroxide solution for at least six hours.

After this normal disinfection cycle, the right and left contact lenses were transferred individually to vials filled with $2 \mathrm{~mL}$ sterile phosphate buffer saline (PBS; pH 7.4 NaCl $8 \mathrm{~g}^{-1}$, $\mathrm{KCl} 0.2 \mathrm{~g}^{-1}, \mathrm{Na}_{2} \mathrm{HPO}_{4}$ $1.15 \mathrm{~g}^{-1}, \mathrm{KH}_{2} \mathrm{PO}_{4} 0.2 \mathrm{~g} \mathrm{1}^{-1}$ ) using sterile forceps. A sterile magnetic stirring bar was added to each vial and vortexed for 10 - 20 seconds, and then 400 $\mu \mathrm{L}$ of the PBS was inoculated onto each of three chocolate blood agar plates (Thermo Fisher Scientific, Waltham, MA, USA) and one Sabouraud's dextrose agar plate (Thermo Fisher Scientific, Waltham, MA, USA). The chocolate blood agar plates were incubated at $37^{\circ} \mathrm{C}$ for 24,48 hours, and 96 hours under aerobic, microaerophilic, and anaerobic conditions, respectively, and the Sab-ouraud's dextrose agar plate was incubated at room temperature for one week for fungal recovery. A total colony count was assessed to determine the number of microbes on each plate. Preliminary identification involved examining colony morphology followed by microscopy and Gram staining (Remel ${ }^{\mathrm{TM}}$ Gram staining kit; Thermo Fisher Scientific, Waltham, MA, USA) for bacteria. Unique colonies were preserved at $-80^{\circ} \mathrm{C}$ for further identification of bacteria using biochemical techniques (Figure 1). ${ }^{36}$
A
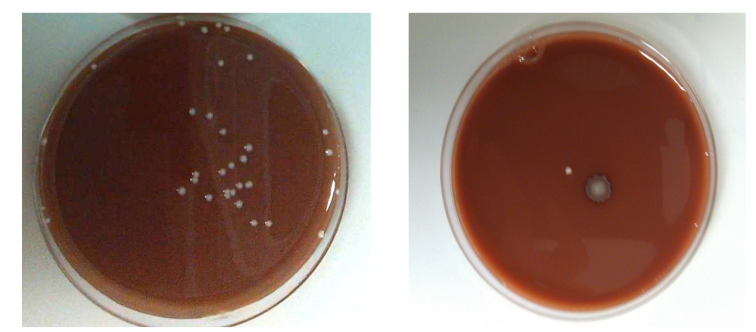

Figure 1. Representative images of bacterial recovery from chocolate agar plates from contaminated (left) soft contact lens and (right) ortho-k storage vials.

The inside of the lens storage vials (well, basket and lid) were swabbed with a sterile cotton swab premoistened with PBS, and then the swab was placed in $2 \mathrm{~mL}$ of PBS and vortexed at $700 \mathrm{~g}$ for 10-20 seconds. The number of bacteria (CFUs) was calculated per millilitre of sample by dividing the number of colonies by dilution. The rest of the bacterial and fungal culture procedures and identification methods were the same as mentioned for contact lenses.

\section{STATISTICAL ANALYSIS}

The number of viable organisms was recorded as colony-forming units (CFUs) per sample and converted into $\log 10 \mathrm{CFU} / \mathrm{mL}$. Data analysis was performed using Microsoft Excel 2010 and Statistical Package for Social Science for Windows version 27.0 (SPSS, Inc, Chicago, IL, USA); significance was considered $\mathrm{P}<$ 0.05 . The Mann-Whitney U test was used to compare the percentage of Gram-positive and Gram-negative bacteria recovered from SCL and ortho-k lenses and storage vials. The RSS scores for contaminated and non-contaminated ortho-k and SCL lenses and storage vials were also compared using the Mann-Whitney $\mathrm{U}$ test. The subjective responses of the RSS scores and the proportion of the microbial contamination of contact lens and storage vials were analysed using the Fischer Exact test.

\section{RESULTS}

Twenty-five participants were enrolled, but one did not meet the study criteria for lens fitting at the baseline exam, one did not have an acceptable lens fit, and two withdrew due to other time commitments. 
A total of 21 participants ( 11 females and 10 males) with a mean age of $25 \pm 5$ years (range: 19 to 38 years inclusive) completed the study (Table 1). All participants previously wore SCL. Participants wore the study contact lenses for an average of $7.5 \pm 1$ hours per night (range: 7 to 9 hours) during ortho-k lens use and $8.2 \pm 2$ hours per day (range: 4.5 to 13 hours) during SCL use.

\section{Contact lens and storage case contamination}

One (5\%) ortho-k lens, five (24\%) ortho-k storage vials, one (5\%) SCL and one (5\%) SCL storage vials were contaminated $(\mathrm{P}>0.05$; Figure $2 \mathrm{~A})$. None of the participants had contamination with both ortho-k and SCL lenses. Only one participant had both lens and storage vial contamination during the use of ortho-k lenses ( $P>0.05$; Figure $2 B)$. Due to the small number of contaminated samples and small subject samples in this pilot study, the contact lens and storage vial contamination was collapsed (e.g., if either the storage vial or lens was contaminated, the sample was considered positive for that lens type). Overall, $24 \%$ of the ortho$\mathrm{k}$ and $10 \%$ of the SCL samples were contaminated (Figure 2A). There was no correlation between gender and the rate of contamination $(\mathrm{P}>0.05)$.

Table 1. Demographic and baseline information $(n=21)$

\begin{tabular}{|c|c|c|c|}
\hline \multirow{2}{*}{\multicolumn{2}{|c|}{ Sex }} & \multirow{2}{*}{$\begin{array}{c}\text { Male } \\
10\end{array}$} & \multirow{2}{*}{$\begin{array}{c}\text { Female } \\
11\end{array}$} \\
\hline & & & \\
\hline Age (years) & Mean \pm SD & $26.3 \pm 4.6$ & $25.0 \pm 5.1$ \\
\hline \multirow{2}{*}{ Ethnicity } & Hispanic or Latino & 1 & 4 \\
\hline & Non-Hispanic & 9 & 7 \\
\hline \multirow{5}{*}{ Race } & White & 3 & 4 \\
\hline & African American & 2 & 2 \\
\hline & Asian & 5 & 4 \\
\hline & Pacific Islander & 0 & 0 \\
\hline & Other & 0 & 1 \\
\hline \multirow{2}{*}{\multicolumn{2}{|c|}{ Baseline information }} & \multicolumn{2}{|c|}{ All participants (21) } \\
\hline & & Range (min-max) & Mean \pm SD \\
\hline \multicolumn{2}{|c|}{ Subjective Refraction Sphere (OD) } & -0.25 to -4.75 & $-2.87 \pm 1.02$ \\
\hline \multicolumn{2}{|c|}{ Subjective Refraction Cylinder (OD) } & -1.25 to -3.00 & $-2.09 \pm 0.52$ \\
\hline \multicolumn{2}{|c|}{ Subjective Refraction Sphere (OS) } & -0.00 to -4.75 & $-2.60 \pm 1.35$ \\
\hline \multicolumn{2}{|c|}{ Subjective Refraction Cylinder (OS) } & -1.25 to -3.00 & $-2.01 \pm 0.49$ \\
\hline
\end{tabular}

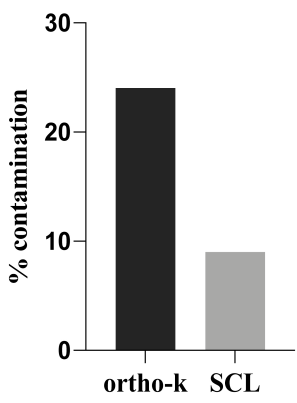

B

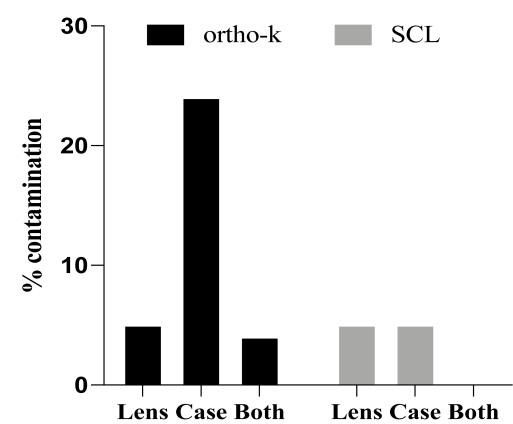

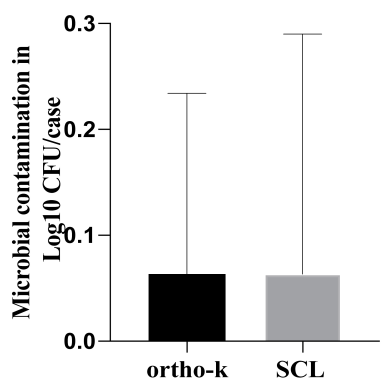

Figure 2. (A) percent of contaminated ortho-k and SCL lenses and storage cases; (B) percent of contaminated SCL and ortho-k lenses and storage vials; (C) the number of microbes recovered from ortho-k and SCL lenses and storage vials. 
There was no difference in the microbial types isolated from SCL and ortho-k contact lenses and storage vials $(\mathrm{P}>0.05)$. All contaminated contact lenses and storage vials were contaminated with Gram-positive bacteria, specifically coagulase-negative staphylococci (CNS). There was no recovery of Gram-negative bacteria from the lenses or storage vials. There was no difference in the number of CFUs cultured after ortho-k (mean 0.10, range: 0-0.66 Log10 CFUs) compared to SCL wear (mean 0.06, range: $0-1.00$ Log10 CFUs) (P > 0.05; Figure 2C).

\section{Ocular signs and symptoms}

Ocular variables were measured for both eyes, but as there were no differences between the eyes upon statistical analysis, data for the left eye are reported. No significant differences were found between participants' eyes using either ortho-k or SCLs for bulbar or palpebral conjunctival redness, palpebral redness, roughness, or conjunctival staining $(\mathrm{P}>$ $0.05)$. Similarly, there was no significant difference in these outcomes concerning contamination of lenses and storage vials $(\mathrm{P}>0.05)$. None of the participants developed any contact lens induced complications during the study period.

Overall, there was no significant difference in discomfort, vision, or lens handling symptoms between contaminated and non-contaminated lenses/storage vials (Figure 3). However, two of the participants

A

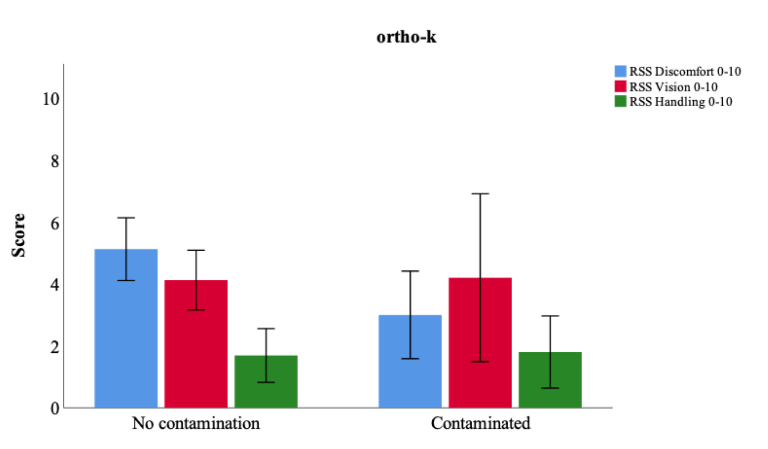

who had contaminated SCL, or storage vials reported watering of the eyes and tiredness during lens wear, although this was not statistically significant (data not shown, $\mathrm{P}>0.05$ ).

\section{DISCUSSION}

The purpose of this study was to prospectively assess the bioburden and efficacy of hydrogen-peroxide solution in disinfecting SCL, and ortho-k lenses and storage vials used by the same group of participants for the same period. In the current study, the overall rate of microbial contamination of contact lens storage lens vials was $30 \%$, which is much lower than one previously reported study with ortho-k lens wear. ${ }^{50}$

The hydrogen-peroxide storage vials used in this study are barrel-shaped. Another study of ortho-k lenses and the combination of MPDS found a higher contamination rate in flat storage cases than barrel cases/vials. ${ }^{51}$ The ability to hold a greater volume of disinfecting solution could be the reason for lower contamination levels with these vials. The lower level of contact lens and storage case contamination could also be due to the hydrogen-peroxide disinfecting system. ${ }^{52}$ To date, most of the previous studies assessed the rate and amount of microbial contamination of contact lenses or storage vials using MPDS. Microbial contamination of contact lens and lens storage cases varies depending upon the cleaning and disinfecting solution, the types of lens materials, and

B

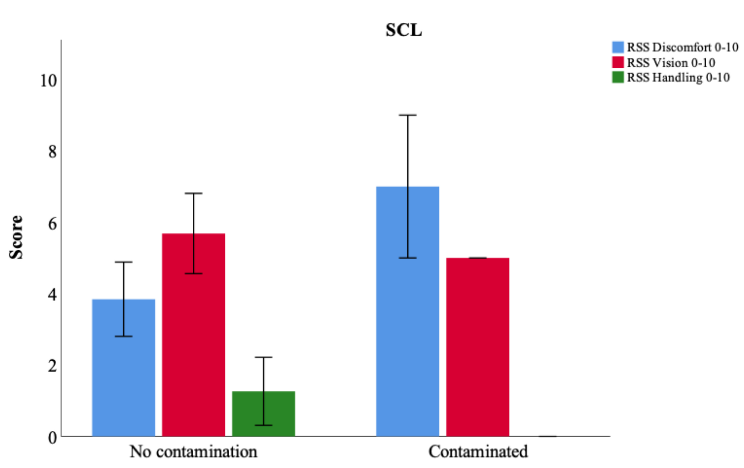

Figure 3. RSS survey scores for comfort, vision, and lens handling with (A) ortho-k and (B) SCL, separated based on contamination. The scoring scale is such that 0 is extremely satisfied with comfort, vision, and handling, and 10 is extremely unsatisfied. 
wear modality (Table 2). ${ }^{34-37}$ The performance of commercially available disinfecting solutions have been compared, and CLEAR CARE ${ }^{\circledR}$ hydrogen-peroxide system was the most effective disinfectant in soft lens wear due to its higher disinfection activity and lower toxicity to ocular cells. ${ }^{53,54}$ Most of the in vivo studies were consistent with the notion that the lower level of microbial recovery during rigid lens wear is partly because of a low incidence of bacterial adherence on rigid lenses. ${ }^{55}$ The contact lens, lens storage case, and ocular surface may offer a suitable environment for the survival of bacteria. Due to its resistance to contact lens care systems, $P$. aeruginosa can adhere and colonize lens materials during use and survive in lens storage cases. ${ }^{56}$ Interestingly, our study did not recover Gram-negative bacteria. The lack of Gram-negative bacteria and the overall reduced level of microbial contamination could be due to the antimicrobial efficacy of the hydrogen-peroxide system, which was also reported in previous studies. ${ }^{57,58}$ Although Gram-negative bacteria, including Pseudomonas, are commonly associated with microbial keratitis, Gram-positive bacteria have been isolated in contact lens related keratitis. Bourcier et al. showed that twothirds of the bacterial keratitis in contact lens wear was associated with Gram-positive bacterial species, including streptococci and staphylococci, particularly S. aureus. ${ }^{59}$

There was only one instance when both the contact lens and vial were found contaminated. This suggests that although lens storage cases may be contaminated, the contact lenses themselves may be sufficiently disinfected. However, future studies should consider the assessment of both contact lenses and lens storage cases to better understand the rates of contact lens compared to case contamination.

The most frequently isolated microbes in this study were CNS. The rate and level of microbial contamination with ortho-k use were higher than with SCL, though not statistically significant. Coagulasenegative staphylococci are one of the most frequently isolated Gram-positive bacteria from conjunctival swab of healthy individuals, with low frequency of isolation of bacteria such as Propionibacterium sp., Staphylococcus aureus, Micrococcus $s p .{ }^{60}$ Similarly, coagulase-negative staphylococci were the most commonly isolated contaminant from lenses and lens storage cases/vials in the daily wear contact lens wearing population. ${ }^{36,38}$ Although rarely, coagulase-negative staphylococci have been associated with contact lens associated corneal infection. However, Stapleton et al. mentioned in a systematic literature review Coagulase-negative staphylococci was the second most commonly isolated microbe from contact lens induced $\mathrm{MK} .{ }^{27} \mathrm{~A}$ previous study showed that the incidence of Gram-positive bacteria increases after eight hours of eye closure, whereas there were no increases in Gram-negative or fungal organisms. ${ }^{61}$ Multiple genera and species are associated with contact lens induced MK and CIEs. ${ }^{8,18}$ Causative microorganisms have been isolated from contact lenses and contact lens related accessories even in asymptomatic wearers (Table 2). The present study also suggests higher contamination of Gram-positive bacteria after overnight (closed eye) ortho-k lens wear.

Differences in contact lens material properties can cause different bacterial adhesion and deposition on lenses that may impact microbial contamination and isolation from lenses or lens cases. In the present study, we used the ortho-k lens material Paragon HDS 100 and omafilcon B soft hydrogel lenses. Soft hydrogel contact lenses deposit more protein compared to silicone hydrogel contact lenses, which deposit more lipid. Zhang et al. reported significant microbe variability between SCL and ortho-k lens wearing groups using operational taxonomic units. ${ }^{62}$ Ortho-k lens wearers had an abundance of Bacillus, Tatumella, and Lactobacillus, while Elizabethkingia was abundant in SCL wearers and Delftia populations reduced in SCL wearers. ${ }^{62}$ Future studies should compare the microbial isolates from overnight wear of ortho-k lenses and silicone hydrogel contact lenses. Moreover, the advanced 16s rRNA bacterial identification technique should be used for more accurate results of bacterial identification.

Using a contact lens and storage case contamination rate of $24 \%$ with the population variance of 1000 , a sample size of 34 would be required (5\% level of significance with $80 \%$ power and $20 \%$ drop out rate) to significant differences in contamination between ortho-k and SCL lens wearers. 


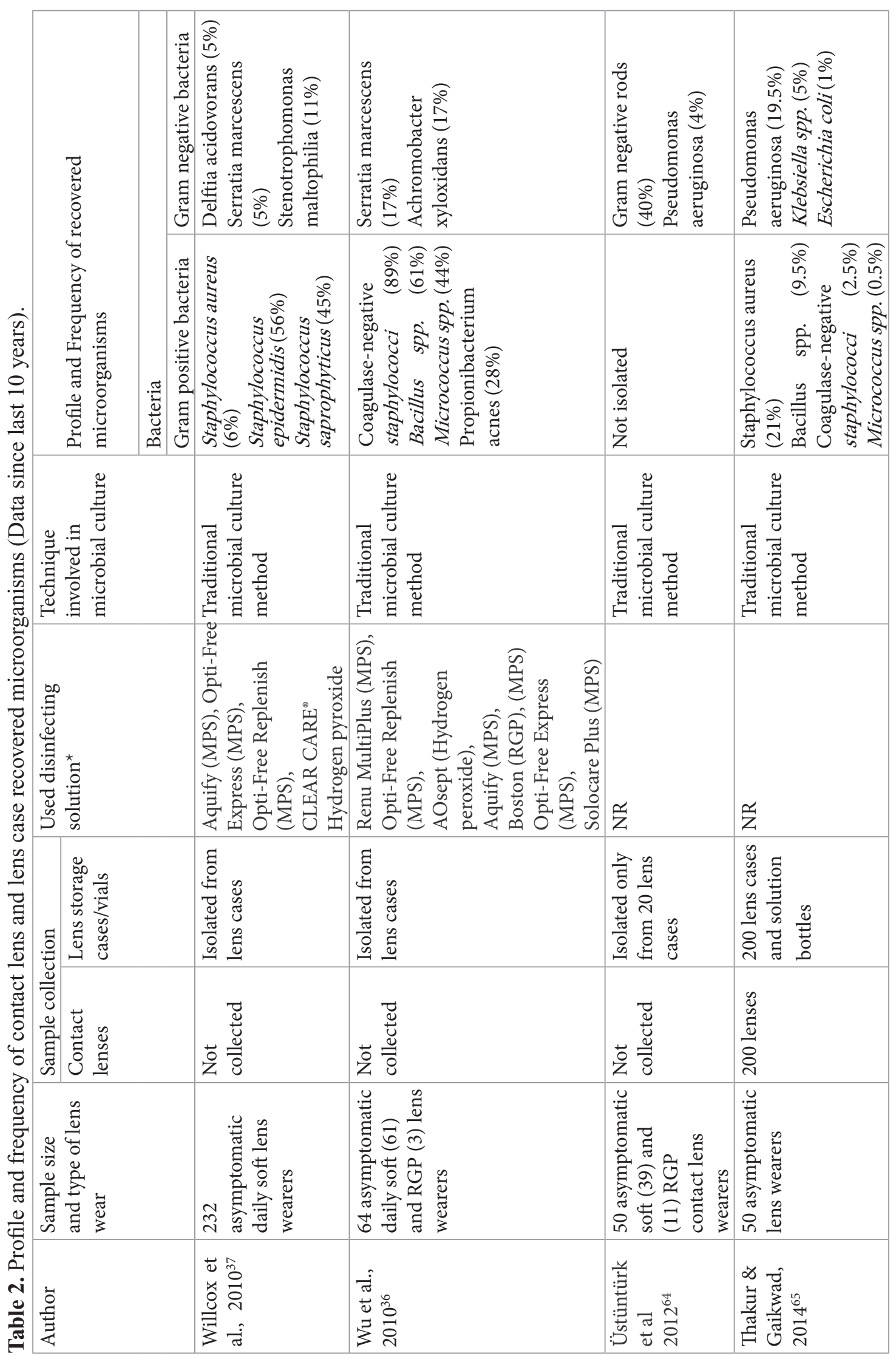

J Cont Lens Res Sci Vol 5(1):e19-e31; October 26, 2021

This article is distributed under the terms of the Creative Commons AttributionNon Commercial 4.0 International License. @Datta et al. 


\begin{tabular}{|c|c|c|c|c|c|}
\hline 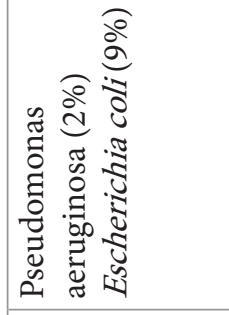 & 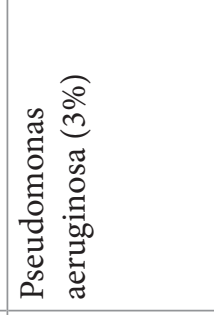 & 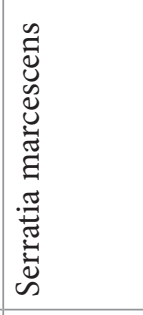 & 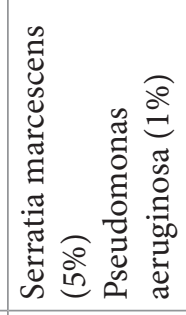 & 占 & 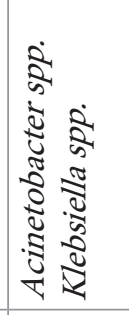 \\
\hline 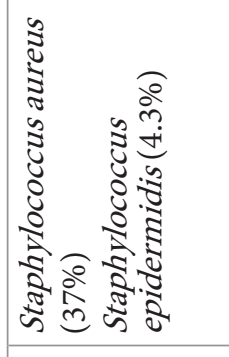 & 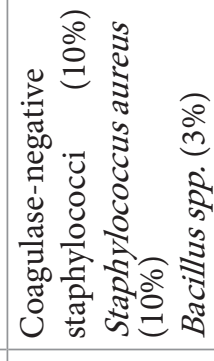 & 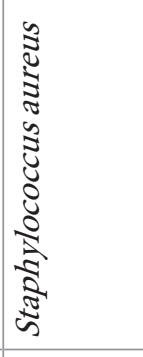 & 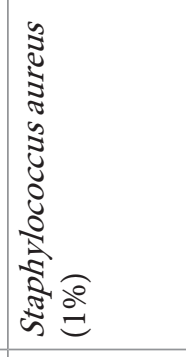 & 号 & 艺 \\
\hline 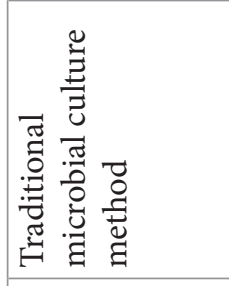 & 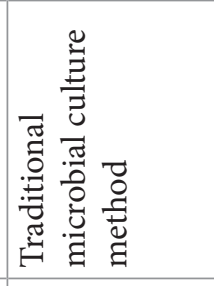 & 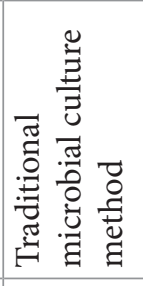 & 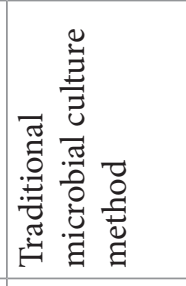 & 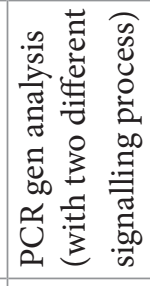 & 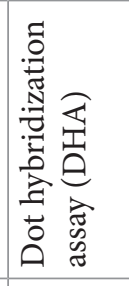 \\
\hline 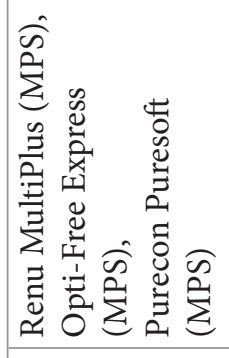 & 号 & 号 & 㟔 & 㟔 & 岩 \\
\hline 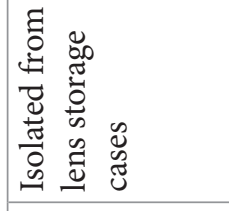 & 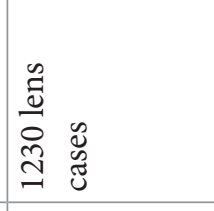 & 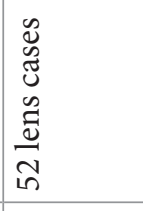 & 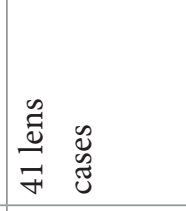 & 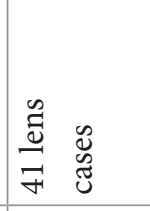 & 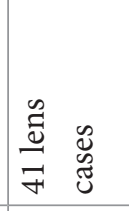 \\
\hline 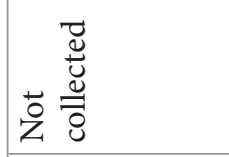 & 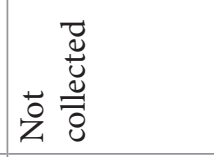 & 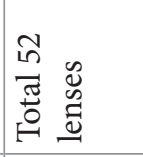 & 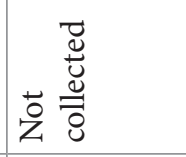 & 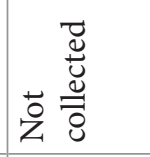 & 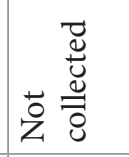 \\
\hline 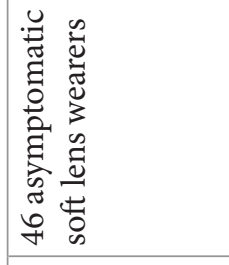 & 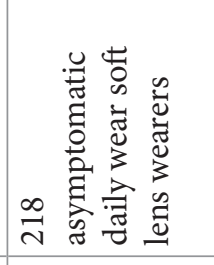 & 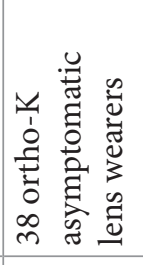 & 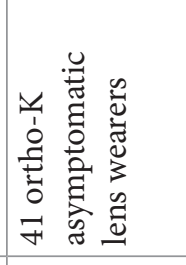 & 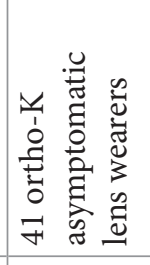 & 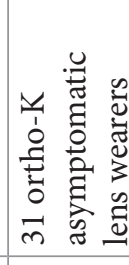 \\
\hline 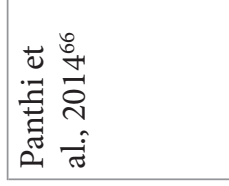 & 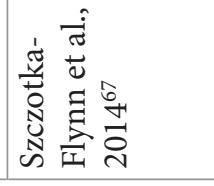 & 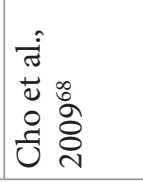 & 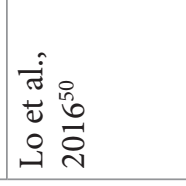 & 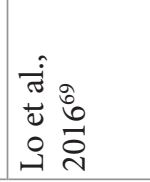 & 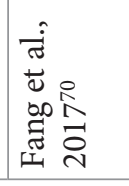 \\
\hline
\end{tabular}

J Cont Lens Res Sci Vol 5(1):e19-e31; October 26, 2021

This article is distributed under the terms of the Creative Commons AttributionNon Commercial 4.0 International License. ODatta et al. 
This study also investigated the correlation between the contact lens and storage vial contamination and the subjective response of contact lens wearing comfort, vision, and handling issues. ${ }^{49}$ While no significant correlation between microbial contamination was identified, some participants with contamination noted symptoms of watery and tired eyes. There was also no association between ocular signs and microbial contamination in this study. Szczotka-Flynn et al. previously reported a positive correlation [adjusted odds ratios (and 95\% confidence intervals); 4.11 (1.17-14.46)] between microbial bioburden of soft silicone hydrogel contact lenses in continuous wearers and ocular discomfort, dryness, redness, and itchiness. ${ }^{63}$ The lack of positive correlation between microbial contamination and signs or symptoms in this study is potentially due to the small sample size and further supports the need for a larger study.

\section{CONCLUSIONS}

In summary, the current study identified differences in contamination rates and levels of contamination during 10 days of SCL and ortho-k lens wear in participants using a hydrogen-peroxide solution. The overall rate of microbial contamination of contact lens storage vials was $30 \%$, which is much lower than one previously reported study with ortho-k lens wear. Therefore, the use of the hydrogen-peroxide solution in ortho-k users and SCL users should be recommended to reduce contact lens contamination. Further research is needed to explore this finding and its associations with clinical signs and symptoms.

\section{DISCLOSURE}

Ananya Datta: none

Erin Tomiyama: none

Kathryn Richdale: Consulting: CooperVision, Paragon, Novartis; Research support: Alcon, Novartis, Paragon, Euclid

\section{Grant information}

- National Institutes of Health Loan Repayment Program (Erin S. Tomiyama)

- NIH P30 EY00755

- Paragon Vision Sciences provided the orthokeratology lenses but had no role in the study design or outcomes.

\section{REFERENCES}

1. Pucker AD, Tichenor AA. A Review of Contact Lens Dropout. Clin Optom. 2020;12:85-94. doi:10.2147/ OPTO.S198637

2. Cope JR, Collier SA, Nethercut H, Jones JM, Yates K, Yoder JS. Risk Behaviors for Contact Lens-Related Eye Infections Among Adults and Adolescents United States, 2016. MMWR Morb Mortal Wkly Rep. 2017;66(32):841. doi:10.15585/MMWR.MM6632A2

3. Chen C, Cheung SW, Cho P. Myopia control using toric orthokeratology (TO-SEE study). Investig Opthalmology Vis Sci. 2013;54(10):6510.

4. Cho P, Cheung S-W. Retardation of myopia in Orthokeratology (ROMIO) study: a 2-year randomized clinical trial. Investig Opthalmology Vis Sci. 2012;53(11):7077.

5. Kakita T, Hiraoka T, Oshika T. Influence of overnight orthokeratology on axial elongation in childhood myopia. Investig Opthalmology Vis Sci. 2011;52(5):2170.

6. Cho P, Cheung W, Edwards M. The longitudinal orthokeratology research in children (LORIC) in Hong Kong: a pilot study on refractive changes and myopic control. Glob Orthokeratology Symp. 2005;30 :71-80.

7. Bullimore MA, Sinnott LT, Jones-Jordan LA. The risk of microbial keratitis with overnight corneal reshaping lenses. Optom Vis Sci. 2013;90(9):937-944.

8. Watt KG, Swarbrick HA. Trends in microbial keratitis associated with orthokeratology. Eye Contact Lens Sci Clin Pract. 2007;33(Supplement):373-377.

9. Hsiao C-H, Lin H-C, Chen Y-F, et al. Infectious keratitis related to overnight orthokeratology. Cornea. 2005;24(7):783-788.

10. Goodlaw E. Risk of infection from sleeping with contact lenses on: causes of risk. Optom Vis Sci. 1996;73(3):156-158.

11. Chan TCY, Li EYM, Wong VWY, Jhanji V. Orthokeratology-associated infectious keratitis in a tertiary care eye hospital in Hong Kong. Am J Ophthalmol. 2014;158(6):1130-1135.e2.

12. Wu Y, Willcox M, Zhu H, Stapleton F. Contact lens hygiene compliance and lens case contamination: A review. Contact Lens Anterior Eye. 2015;38(5):307-316.

13. Sankaridurg PR, Sweeney DF, Sharma S, et al. Adverse events with extended wear of disposable hydrogels: Results for the first 13 months of lens wear. Ophthalmology. 1999;106(9):1671-1680. doi:10.1016/ S0161-6420(99)90346-9 
14. Young AL, Leung ATS, Cheng LL, Law RWK, Wong AKK, Lam DSC. Orthokeratology Lens-Related corneal ulcers in children: A case series. Ophthalmology. 2004;111(3):590-595. doi:10.1016/J.OPHTHA.2003.06.003

15. Willcox MD, Holden BA. Contact lens related corneal infections. Biosci Rep. 2001;21(4):445-461.

16. Stapleton F, Keay L, Edwards K, et al. The incidence of contact lens-related microbial keratitis in Australia. Ophthalmology. 2008;115(10):1655-1662.

17. Wihelmus K. Review of clinical exeperience with microbial keratitis associated with contact lenses. CLAO. 1987;13:211-214.

18. Watt K, Swarbrick HA. Microbial keratitis in overnight orthokeratology: review of the first 50 cases. Eye Contact Lens. 2005;31(5):201-208.

19. Szczotka-Flynn L, Lass JH, Sethi A, et al. Risk factors for corneal infiltrative events during continuous wear of silicone hydrogel contact lenses. Invest Ophthalmol Vis Sci. 2010;51(11):5421-5430.

20. Sweeney DF, Jalbert I, Covey M, et al. Clinical characterization of corneal infiltrative events observed with soft contact lens wear. Cornea. 2003;22(5):435-442.

21. Nilsson SE. Seven-day extended wear and 30-day continuous wear of high oxygen transmissibility soft silicone hydrogel contact lenses: a randomized 1-year study of 504 patients. CLAO. 2001;27(3):125-136.

22. Sankaridurg PR, Sharma S, Willcox M, et al. Bacterial colonization of disposable soft contact lenses is greater during corneal infiltrative events than during asymptomatic extended lens wear. J Clin Microbiol. 2000;38(12):4420-4424.

23. Sankaridurg PR, Willcox MDP, Sharma S, et al. Haemophilus influenzae adherent to contact lenses associated with production of acute ocular inflammation. J Clin Microbiol. 1996;34(10):2426-2431.

24. Szczotka-Flynn L, Chalmers R. Incidence and epidemiologic associations of corneal infiltrates with silicone hydrogel contact lenses. Eye Contact Lens. 2013;39(1):48-52.

25. Donzis P, Mondino P, Weissman B, Bruckner D. Microbial contamination of contact lens care systems. Am J Ophthalmol. 1987;104:325-333.

26. Wilson LA, Sawant AD, Simmons RB, Ahearn DG. Microbial contamination of contact lens storage cases and solutions. Am J Ophthalmol. 1990;110(2):193-198.

27. Stapleton F, Keay L, Sanfilippo P, Katiyar S, Edwards K, Naduvilath T. Relationship between climate, disease severity and causative organism for contact lens-associated microbial keratitis in Australia. Am J Ophthalmol. 2007;144(5):690-698.e1.

28. Galentine PG, Cohen EJ, Laibson PR, Adams CP, Michaud $\mathrm{R}$, Arentsen JJ. Corneal ulcers associated with contact lens wear. Arch Ophthalmol. 1984;102(6):891-894.

29. Bennett HGB, Hay J, Kirkness CM, Seal D V, Devonshire P. Antimicrobial management of presumed microbial keratitis: guidelines for treatment of central and peripheral ulcers. Br J Ophthalmol. 1998;82(2):137-145.

30. Cheng KH, Leung SL, Hoekman HW, et al. Incidence of contact-lens-associated microbial keratitis and its related morbidity. Lancet. 1999;354(9174):181-185.

31. Mubareka S, Alfa M, Harding GK, Booton G, Ekins M, Vancaeseele P. Acanthamoeba species keratitis in a soft contact lens wearer molecularly linked to well water. Can J Infect Dis Med Microbiol. 2006;17(2):120-122.

32. Wilhelmus K, Robinson N, Font R, Hamill M, Jones D. Fungal keratitis in contact lens wearers. Am J Ophthalmol. 1988;106(6):708-714.

33. McLaughlin-Borlace L, Stapleton F, Matheson M, Dart JK. Bacterial biofilm on contact lenses and lens storage cases in wearers with microbial keratitis. J Appl Microbiol. 1998;84(5):827-838.

34. Devonshire P, Munro FA, Abernethy C, Clark BJ. Microbial contamination of contact lens cases in the west of Scotland. Br J Ophthalmol. 1993;77(1) :41-45.

35. Gopinathan U, Stapleton F, Sharma S, et al. Microbial contamination of hydrogel contact lenses. J Appl Microbiol. 1997;82(5):653-658.

36. Wu Y, Zhu H, Harmis N, Iskandar S, Willcox M, Stapleton F. Profile and frequency of microbial contamination of contact lens cases. Optom Vis Sci. 2010;87(3):152-158.

37. Willcox MDP, Carnt N, Diec J, et al. Contact lens case contamination during daily wear of silicone hydrogels. Optom Vis Sci. 2010;87(7):456-464.

38. Szczotka-Flynn LB, Pearlman E, Ghannoum M. Microbial contamination of contact lenses, lens care solutions, and their accessories: a literature review. Eye Contact Lens. 2010;36(2):116-129.

39. Tomiyama ES, Hu C, Marsack JD, Richdale K. Greater higher order aberrations induced by toric orthokeratology versus soft toric multifocal contact lens wear. Ophthalmic Physiol Opt. 2021;41(4):726-735. doi:10.1111/ OPO.12839

40. Dantam J, Zhu H, Willcox M, et al. In vivo assessment of antimicrobial efficacy of silver-impregnated

J Cont Lens Res Sci Vol 5(1):e19-e31; October 26, 2021

This article is distributed under the terms of the Creative Commons AttributionNon Commercial 4.0 International License. CDatta et al. 
contact lens storage cases. Invest Ophthalmol Vis Sci. 2012;53(3):1641-1648.

41. Kanpolat A, Kalayci D, Arman D, Drunk K. Contamination in contact lens care system. CLAO. 1992;18(2):104-107.

42. Midelfart J, Midelfart A, Bevanger L. Microbial contamination of contact lens cases among mediacal students. CLAO. 1996;22(1):21-24.

43. Datta A, Willcox MDP, Stapleton F. In vivo efficacy of silver-impregnated barrel contact lens storage cases. Contact Lens Anterior Eye. 2021;44(4):101357. doi:10.1016/J.CLAE.2020.08.001

44. Yung AMS, Boost M V, Cho P, Yap M. The effect of a compliance enhancement strategy (self-review) on the level of lens care compliance and contamination of contact lenses and lens care accessories. Clin Exp Optom. 2007;90(3):190-202.

45. Boost M V, Cho P, Hong T, Polytechnic K, Special HK. Microbial flora of tears of orthokeratology patients, and microbial contamination of contact lenses and contact lens accessories. Optom Vis Sci. 2005;82(6) :451-458.

46. Pens CJ, Costa M da, Fadanelli C, Caumo K, Rott M. Acanthamoeba spp. and bacterial contamination in contact lens storage cases and the relationship to user profiles. Parasitol Res. 2008;103(6):1241-1245.

47. Gray TB, Cursons RT, Sherwan JF, Rose PR. Acanthamoeba, bacterial, and fungal contamination of contact lens storage cases. Br J Ophthalmol. 1995;79(6) :601-605.

48. Paragon Vision Sciences Inc. Professional fitting Paragon CRT $₫$ Paragon CRT $® 100$ Contact lens corneal refractive therapy. 2015:1-16.

49. Diec J, Naduvilath T, Tilia D, Papas EB, Lazon de la Jara P. Discrimination of subjective responses between contact lenses with a novel questionnaire. Contact Lens Anterior Eye. 2017;40(6):367-381.

50. Lo J, Kuo M-T, Chien C-C, Tseng S-L, Lai Y-H, Fang P-C. Microbial bioburden of orthokeratology contact lens care system. Eye Contact Lens Sci Clin Pract. 2016;42(1):61-67.

51. Wanga J, Liua L, Boostb M, Yapb M, Cho P. Risk factors associated with contamination of orthokeratology lens cases. Contact Lens Anterior Eye. 2020;43:178-184.

52. Gabriel MM, McAnally C, Bartell J, et al. Biocidal Efficacy of a Hydrogen Peroxide Lens Care Solution Incorporating a Novel Wetting Agent. Eye Contact Lens. 2019;45(3):164-170.
53. Willcox M. Hydrogen peroxide versus multipurpose disinfecting solutions - what are the differences? Ocul Surf Charact Asian Eye. 2008.

54. Papas E, Carnt N, Willcox M, Holden B. Complications associated with care product use during silicone daily wear of hydrogel contact lens. Eye Contact Lens. 2008;33(6):392-393.

55. Hovding $\mathrm{G}$. The conjunctival and contact lens bacterial flora during lens wear. Acta Ophthalmol. 1981;59(3):387401. doi:10.1111/j.1755-3768.1981.tb03004.x

56. Lakkis C, Fleiszig SMJ. Resistance of Pseudomonas aeruginosa isolates to hydrogel contact lens disinfection correlates with cytotoxic activity. J Clin Microbiol. 2001;39(4):1477-1486.

57. Gabriel MM, McAnally C, Chen H, Srinivasan S, Manoj V, Garofalo R. Hydrogen Peroxide Disinfecting Solution for Gas Permeable Contact Lenses: A Review of the Antimicrobial Efficacy, Compatibility, and Safety Performance of a One-Step Lens Care System. Clin Optom. 2021;13:7. doi:10.2147/OPTO.S280046

58. MM G, C M, J B, et al. Biocidal Efficacy of a Hydrogen Peroxide Lens Care Solution Incorporating a Novel Wetting Agent. Eye Contact Lens. 2019;45(3):164-170. doi:10.1097/ICL.0000000000000549

59. Bourcier T, Thomas F, Borderie V, Chaumeil C, Laroche L. Bacterial keratitis: predisposing factors, clinical and microbiological review of 300 cases. Br J Ophthalmol. 2003;87(7):834-839.

60. Willcox MDP. Characterization of the normal microbiota of the ocular surface. Exp Eye Res. 2013;117:99-105. doi:10.1016/J.EXER.2013.06.003

61. Ramachandran L, Sharma S, Sankaridurg PR, et al. Examination of the conjunctival microbiota after 8 hours of eye closure. CLAO J. 1995;21(3):195-199.

62. Zhang H, Zhao F, Hutchinson DS, et al. Conjunctival Microbiome Changes Associated With Soft Contact Lens and Orthokeratology Lens Wearing. Invest Ophthalmol Vis Sci. 2017;58(1):128-136. doi:10.1167/ IOVS.16-20231

63. Szczotka-Flynn LB, Bajaksouzian S, Jacobs MR, Rimm A. Risk Factors for contact lens bacterial contamination during continuous wear. Optom Vis Sci. 2009;86(11):1216-1226.

64. Üstüntürk M, Zeybek Z. Microbial contamination of contact lens storage cases and domestic tap water of contact lens wearers. Wien Klin Wochenschr. 2012;124(S3):17-22.

J Cont Lens Res Sci Vol 5(1):e19-e31; October 26, 2021

This article is distributed under the terms of the Creative Commons Attribution-

Non Commercial 4.0 International License. CDatta et al. 
65. Thakur D V, Gaikwad UN. Microbial contamination of soft contact lenses \& accessories in asymptomatic contact lens users. Indian J Med Res. 2014;140(2) :307-309.

66. Panthi S, Paudel P, Chaudhary M, Sapkota K, Shah DN. Microbial contamination of contact lens care accessories and compliance with care regimens in Nepal. Contact Lens Anterior Eye. 2014;37(1):2-10.

67. Szczotka-Flynn L, Jiang Y, Raghupathy S, et al. Corneal inflammatory events with daily silicone hydrogel lens wear. Optom Vis Sci. 2014;91(1):3-12.
68. Cho P, Boost M, Cheng R. Non-compliance and microbial contamination in orthokeratology. Optom Vis Sci. 2009;86(11):1227-1234.

69. Lo J, Fang P, Chien C, Hsiao C, Tseng S, Lai Y. PCR analysis for assessment of bacterial bioburden in orthokeratology lens cases. 2016;(August 2015):1-8.

70. Fang PC, Lo J, Chang TC, et al. Bacterial bioburden decrease in orthokeratology lens storage cases after forewarning: Assessment by the DNA Dot Hybridization Assay. Eye Contact Lens. 2017;43(3):174-180. doi:10.1097/ICL.0000000000000252 